\title{
Speckled lentiginous nevus: sometimes, but not always, part of a syndrome
}

\author{
Daniele Torchia $\cdot$ Lawrence A. Schachner
}

Received: 5 January 2011 / Accepted: 21 July 2011 / Published online: 2 November 2011

(C) Springer-Verlag 2011

\section{Dear Editor}

We read with great interest the recent paper by Boncoraglio et al. [1]. They reported on an adult female patient affected by a large speckled lentiginous nevus (SLN) associated with an intracranial cavernoma and concluded that she had either phacomatosis pigmentovascularis or SLN syndrome [1]. We believe that such a differential diagnosis, and the fact that SLN was part of a syndrome, may not be correct. First, according to current knowledge, the only type of phacomatosis pigmentovascularis featuring a SLN is phacomatosis spilorosea, which is characterized by the association of a macular SLN with nevus roseus [2]. Instead, in the case by Boncoraglio et al., no nevus roseus was present [1]. Second, SLN syndrome is characterized by a papular SLN associated with ipsilateral peripheral nerve, neuromuscular and/or central nervous system disturbances [3]. On the other hand, Boncoraglio et al. did not describe any of these anomalies if we exclude those mechanically induced by the intracranial cavernoma or its surgical management [1]. Third, the only other known syndrome featuring a SLN, i.e., phacomatosis pigmentokeratotica, had to be ruled out because of the lack of an associated nevus sebaceus.

The fact that the intracranial cavernoma was ipsilateral and spacially close to the SLN may suggest that both lesions were determined by the migration of abnormal cell clones at some point during ontogenesis and thus were somehow pathogenically related. However, we recently carried out a systematic review on segmental SLN (unpublished data) and found that only 84 out of the 281 cases reported in the literature $(29.9 \%)$ were part of a syndrome, and only $2(0.7 \%)$ were associated with angiomas (and cutaneous ones) [4, 5] and that in the vast majority of syndromic cases, both SLN and, if present, the associated nevus (nevus roseus or nevus sebaceus) were extensive and often involving both sides of the body.

On the basis of these arguments, it is our opinion that there is not enough ground to call for a (new) syndrome and therefore, that Boncoraglio et al. dealt with an isolated maculopapular SLN randomly associated with intracranial cavernoma.

\section{References}

1. Boncoraglio GB, Parati EA, Ciceri E, Rinaldi R, Capella GL (2010) Intracranial cavernoma and speckled lentiginous nevus: extending the spectrum of phakomatoses? Neurol Sci 31:841-844

2. Happle R (2005) Phacomatosis pigmentovascularis revisited and reclassified. Arch Dermatol 141:385-388

3. Torchia D (2010) More on speckled lentiginous nevus syndrome. Dermatology 221:324-325

4. Brufau C, Moran M, Armijo M (1986) Naevus sur naevus: A propos de 7 observations, trois associees a d'autres dysplasies, et une a un melanome malin invasif. Ann Dermatol Venereol 113:409-418

5. Goldberg LH, Collins SA, Siegel DM (1987) The epidermal nevus syndrome: case report and review. Pediatr Dermatol 4:27-33

D. Torchia $(\bowtie)$ L. A. Schachner

Department of Dermatology and Cutaneous Surgery,

University of Miami Miller School of Medicine,

1295 NW 14th Street, South Bldg, Suite K, Miami,

FL 33125, USA

e-mail: daniele.torchia@unifi.it 